\title{
Amplitude-Phase Synchronization at the Onset of Permanent Spatiotemporal Chaos
}

\author{
Abraham C.-L. Chian, ${ }^{1,2,3, *}$ Rodrigo A. Miranda, ${ }^{1,2,3}$ Erico L. Rempel, ${ }^{2}$ Yoshitaka Saiki, ${ }^{3,4}$ and Michio Yamada ${ }^{3}$ \\ ${ }^{1}$ National Institute for Space Research (INPE) and World Institute for Space Environment Research (WISER), \\ Post Office Box 515, São José dos Campos-SP 12227-010, Brazil \\ ${ }^{2}$ Institute of Aeronautical Technology (ITA) and World Institute for Space Environment Research (WISER), CTA/ITA/IEFM, \\ São José dos Campos-SP 12228-900, Brazil \\ ${ }^{3}$ Research Institute for Mathematical Sciences (RIMS), Kyoto University, Kyoto 606-8502, Japan \\ ${ }^{4}$ Department of Mathematics, Hokkaido University, Hokkaido 060-0810, Japan
}

(Received 23 April 2010; published 25 June 2010)

\begin{abstract}
Amplitude and phase synchronization due to multiscale interactions in chaotic saddles at the onset of permanent spatiotemporal chaos is analyzed using the Fourier-Lyapunov representation. By computing the power-phase spectral entropy and the time-averaged power-phase spectra, we show that the laminar (bursty) states in the on-off spatiotemporal intermittency correspond, respectively, to the nonattracting coherent structures with higher (lower) degrees of amplitude-phase synchronization across spatial scales.
\end{abstract}

Chaotic saddles are nonattracting coherent structures responsible for temporary chaos ubiquitous in spatially extended systems such as the advection-reaction-diffusion systems, excitable media, complex networks, and turbulent shear flows [1]. Experimental evidence of chaotic saddles has been obtained in lasers [2] and pipe flows [3]. Numerical simulations of turbulent shear flows have observed a turbulent flow behaving as a chaotic saddle coexisting with a laminar flow separated by an edge of chaos [4]. The transition to permanent spatiotemporal chaos (STC) via quasiperiodicity is of general interest in nonlinear problems such as drift waves in plasmas, cardiac cells, and ionization fronts [5]. Before the onset of permanent STC, the system is regular in space; at the onset of permanent STC, the spatial order is destroyed. Temporally and spatiotemporally chaotic saddles are the origin of transient and intermittent dynamics of the resulting spatiotemporal intermittency [6].

In this Letter, we investigate the physical mechanism of on-off intermittency at the onset of permanent STC and show that it arises from the amplitude and phase synchronization associated with multiscale interactions in chaotic saddles embedded in the chaotic attractor. The analysis of amplitude-phase synchronization is performed using both Fourier and Lyapunov representations. In particular, we identify the signature of blowout bifurcation typical of on-off intermittency at the transition from the temporally chaotic attractor to the spatiotemporally chaotic attractor.

The regularized long-wave equation that describes nonlinear long-waves in a dispersive medium such as channel flow and seashore, and nonlinear drift waves in magnetized plasmas [6-9], is given by

$\partial_{t} u+c \partial_{x} u+f u \partial_{x} u+a \partial_{t x x} u=-\nu u-\varepsilon \sin (\kappa x-\Omega t)$,

where $\varepsilon$ is the driver amplitude, $c=1, f=-6, a=$
$-0.287, \nu=0.1, \kappa=1$, and $\Omega=0.65[6,8,9]$. Periodic boundary conditions are imposed, $u(x, t)=u(x+2 \pi, t)$. Equation (1) is solved numerically using the pseudospectral method by expanding the wave variable $u(x, t)$ in a Fourier series $u(x, t)=\sum_{k=-N}^{N} \hat{u}_{k}(t) \exp (i k x)$, where $\hat{u}_{k}(t)=(1 / N) \sum_{k=-N}^{N} u(x, t) \exp (-i k x)$ denotes the complex Fourier coefficients, $k$ is normalized as $k=2 \pi n / L$, $n=-N, \ldots, N$, and $L=2 \pi$. Since $u(x, t)$ is a real function, $\hat{u}_{-k}(t)=\hat{u}_{k}(t)$, only $k>0$ need to be considered. We set $N=32$. For each Fourier coefficient, we define its amplitude $\left|\hat{u}_{k}(t)\right|=\sqrt{\hat{u}_{k}(t) \hat{u}_{k}^{*}(t)}$ and phase $\phi_{k}(t)=$ $\arctan \left\{\operatorname{Im}\left[\hat{u}_{k}(t)\right] / \operatorname{Re}\left[\hat{u}_{k}(t)\right]\right\}$. In the parameter regime with which we are working, this definition can capture the difference between the degree of phase synchronization of temporally and spatiotemporally chaotic saddles. In general, a more rigorous definition such as the phase based on the curvature of an arbitrary trajectory must be used [10]. To avoid aliasing numerical errors, only 20 Fourier modes are kept. In this Letter, we study multiscale interactions resulting from nonlinear coupling across spatial scales represented by Fourier modes.

As the control parameter $\varepsilon$ is varied, Eq. (1) exhibits a transition from a spatially regular pattern to permanent STC via the Ruelle-Takens route [5,6]. At the onset of permanent STC, the time series of energy $E(t)=$ $\int_{0}^{2 \pi}\left[u(t)^{2}-a u_{x}(t)^{2}\right] d x / 4 \pi$ displays on-off intermittency shown in the upper panel of Fig. 1, with alternating regime switching from the laminar (on) state to the bursty (off) state. In the Fourier representation, we quantify the degree of amplitude synchronization related to multiscale interactions by the Fourier power spectral entropy $S_{k}^{A}(t)=$ $-\sum_{k=1}^{N} p\left(\hat{u}_{k}(t)\right) \ln \left[p\left(\hat{u}_{k}(t)\right)\right]$, where $p\left(\hat{u}_{k}(t)\right)=\left|\hat{u}_{k}(t)\right|^{2} /$ $\sum_{k=1}^{N}\left|\hat{u}_{k}(t)\right|^{2}$ is the relative weight of the Fourier mode $k$ [6]. The middle panel of Fig. 1 shows the time series of $S_{k}^{A}(t)$. The degree of phase synchronization due to multiscale interactions can be quantified by the Fourier phase 


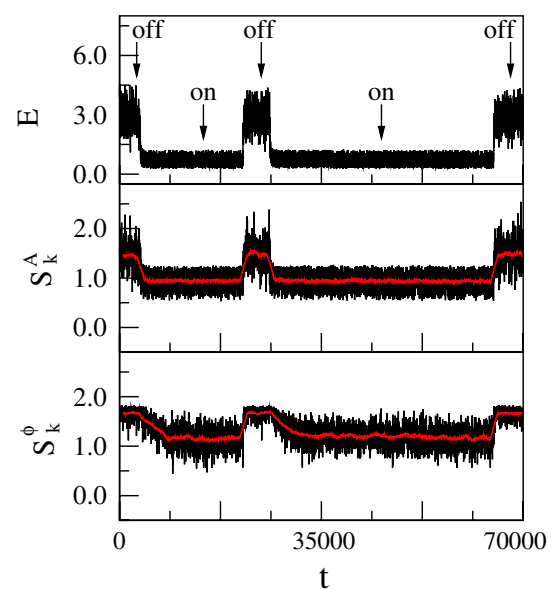

FIG. 1 (color online). Time series of $E, S_{k}^{A}$, and $S_{k}^{\phi}$ for on-off spatiotemporal intermittency at $\varepsilon=0.20005$. The red lines denote averaged curves.

spectral entropy $S_{k}^{\phi}(t)=-\sum_{k=1}^{N} P\left(\delta \phi_{k}(t)\right) \ln \left[P\left(\delta \phi_{k}(t)\right)\right]$, where $P$ denotes the probability distribution function of the Fourier phase differences $\delta \phi_{k}(t)=\phi_{k+1}(t)-\phi_{k}(t)$ [11] and can be determined by constructing a normalized histogram of phase differences $P\left(\delta \phi_{k}\right)=n_{i} / N$, where $n_{i}$ is the number of times $\delta \phi_{k}$ falls into the $i$ th bin of the distribution. The lower panel of Fig. 1 shows the time series of $S_{k}^{\phi}(t)$. In Fig. 1 the gray (red) lines denote time averaging over $t=1256$ to facilitate the visualization of the on-off states. The duality of amplitude and phase synchronization in the on-off spatiotemporal intermittency, confirmed by $S_{k}^{A}(t)$ and $S_{k}^{\phi}(t)$, is demonstrated in Fig. 1. The aims of this Letter are twofold: to probe the origin of this duality and the nature of amplitude-phase synchronization in the on-off spatiotemporal intermittency.

It has been shown that the on-off states in the time series of spatiotemporal intermittency at the onset of permanent STC are linked to chaotic saddles embedded in the chaotic attractor [6]. We choose a Poincaré map defined by $\operatorname{Re}\left\{\hat{u}_{1}(t)\right\}=0$ and $d \operatorname{Re}\left\{\hat{u}_{1}(t)\right\} / d t>0$, then apply the stagger-and-step method [12] to find chaotic saddles, before and after the transition to permanent STC. Figure 2 shows a three-dimensional projection of the Poincare map defined by $\left(\operatorname{Re}\left\{\hat{u}_{2}\right\}, \operatorname{Im}\left\{\hat{u}_{2}\right\}, \operatorname{Re}\left\{\hat{u}_{3}\right\}\right)$. At $\varepsilon=0.199$, before the transition to a spatiotemporally chaotic attractor (STCA), there is a spatiotemporally chaotic saddle (STCS) shown in Fig. 2(a) which is responsible for transient dynamics before the trajectory converges asymptotically to a temporally chaotic attractor (TCA). The TCA is of small size and localized in a sheetlike phase space, as shown in Fig. 2(b). In contrast, the STCS in Fig. 2(a) is of large size and fills up an extended spherelike phase space. At $\varepsilon \sim 0.2$, a crisis [9] occurs whereby TCA loses its stability and is converted to a temporally chaotic saddle (TCS), as seen in Fig. 2(c) for $\varepsilon=0.20005$; STCS is robust and persists after crisis, also superposed in Fig. 2(c).
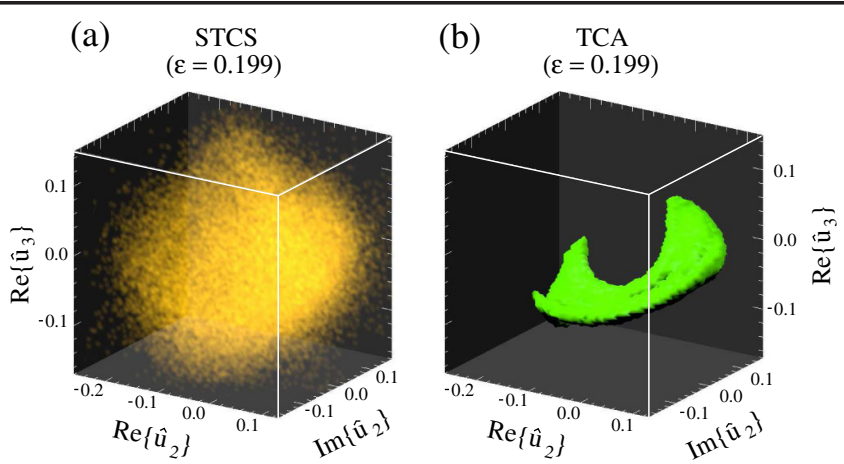

(c) $\mathrm{STCS}+\mathrm{TCS}$
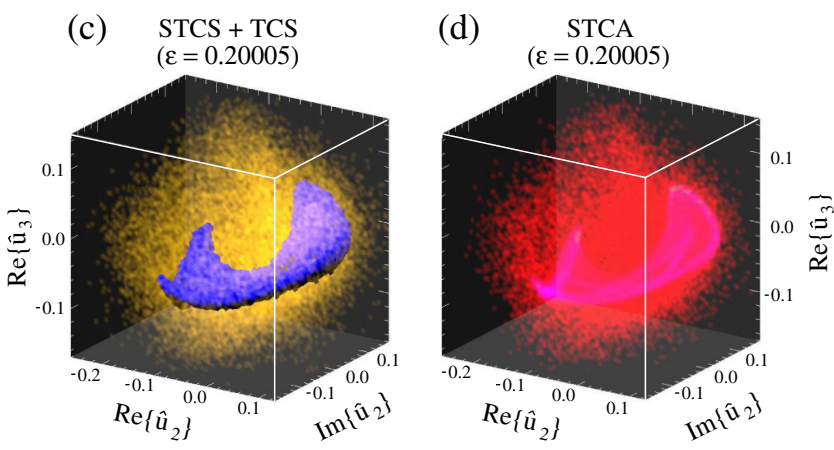

FIG. 2 (color online). Poincaré plots of (a) STCS at $\varepsilon=0.199$, (b) TCA at $\varepsilon=0.199$, (c) STCS and TCS at $\varepsilon=0.20005$, (d) STCA at $\varepsilon=0.20005$.

At the onset of permanent spatiotemporal chaos, TCS becomes coupled to STCS through the coupling unstable periodic orbits to form a STCA, as seen in Fig. 2(d), which occupies almost the same region of phase space as the precrisis STCS and TCA. The postcrisis dynamical system has the ability to keep the memory of its precrisis structures, as evidenced in the postcrisis STCA wherein the phase-space structure of the precrisis sheetlike TCA can still be recognized, which corresponds to TCS of Fig. 2(c). The STCA of Fig. 2(d) corresponds to the time series of the on-off spatiotemporal intermittency of Fig. 1, and TCS (STCS) in Fig. 2(c) corresponds to the on state (off state) of Fig. 1. Figures 2(b) and 2(d) indicate the occurrence of a blowout bifurcation [13] at the transition from TCA to STCA, and an inverse blowout bifurcation at the transition from STCA to TCA. Prior to the blowout bifurcation, trajectories of TCA are confined to a synchronization manifold represented by the sheetlike structure of Fig. 2(b), which is transversely stable. At the onset of the blowout bifurcation, trajectories near this sheetlike structure lose their transverse stability owing to the unstable periodic orbits in STCS and traverse across the sheetlike region, permeating the spherelike phase space seen in 2(d).

Next, we employ the Lyapunov spectrum to characterize the chaotic properties of motion [14], based on the Lyapunov vectors $\delta \mathbf{u}_{j}(t), j=1, \ldots, 2 N$, which denote linear perturbations of the real vector $\mathbf{u}(t)=$ $\left(u_{1}^{R}(t), u_{1}^{I}(t), \ldots, u_{N}^{R}(t), u_{N}^{I}(t)\right)$ constructed from the real and imaginary parts of the complex Fourier coefficients 
$\hat{u}_{k}(t)=u_{k}^{R}(t)+i u_{k}^{I}(t)$. The Lyapunov exponents are determined by the method of Gram-Schmidt orthogonalization and ordered as $\lambda_{j} \geq \lambda_{j+1}$. Since we keep $N=20$ Fourier $(k)$ modes, the number of Lyapunov $(j)$ modes is $2 N=40$. The positive Lyapunov exponents of chaotic attractors and chaotic saddles, before $(\varepsilon=0.199)$ and after $(\varepsilon=0.21)$ the onset of permanent STC, are plotted in Fig. 3(a), normalized by $H=\sum_{j=1}^{q} \lambda_{j}\left(\lambda_{q}>0, \lambda_{q+1} \leq 0\right)$, which is known to be an upper bound or close to the Kolmogorov-Sinai entropy. For TCA before the transition and TCS after the transition, only one Lyapunov vector has a positive Lyapunov exponent, whereas for STCS before the transition, and STCA and STCS after the transition, 14 Lyapunov vectors have positive Lyapunov exponents. Figure 3(a) shows that the Lyapunov spectrum of STCS before the transition is very close to the Lyapunov spectrum of STCA (and STCS) after the transition, which confirms that the precrisis STCS can be used to predict the postcrisis dynamics of STCA [6].

In the Fourier-Lyapunov representation, we quantify the degree of amplitude and phase synchronization due to multiscale $(k, j)$ interactions, respectively, by the timeaveraged Lyapunov power spectral entropy $\left\langle S_{j}^{A}(t)\right\rangle=$ $\left\langle-\sum_{k=1}^{N} p\left(\delta \hat{u}_{k}^{j}(t)\right) \ln \left[p\left(\delta \hat{u}_{k}^{j}(t)\right)\right]\right\rangle$, and the time-averaged Lyapunov phase spectral entropy $\left\langle S_{j}^{\phi}(t)\right\rangle=$ $\left\langle-\sum_{k=1}^{N} P\left(\delta \phi_{k}^{j}(t)\right) \ln \left[P\left(\delta \phi_{k}^{j}(t)\right)\right]\right\rangle$, where \langle\rangle represent
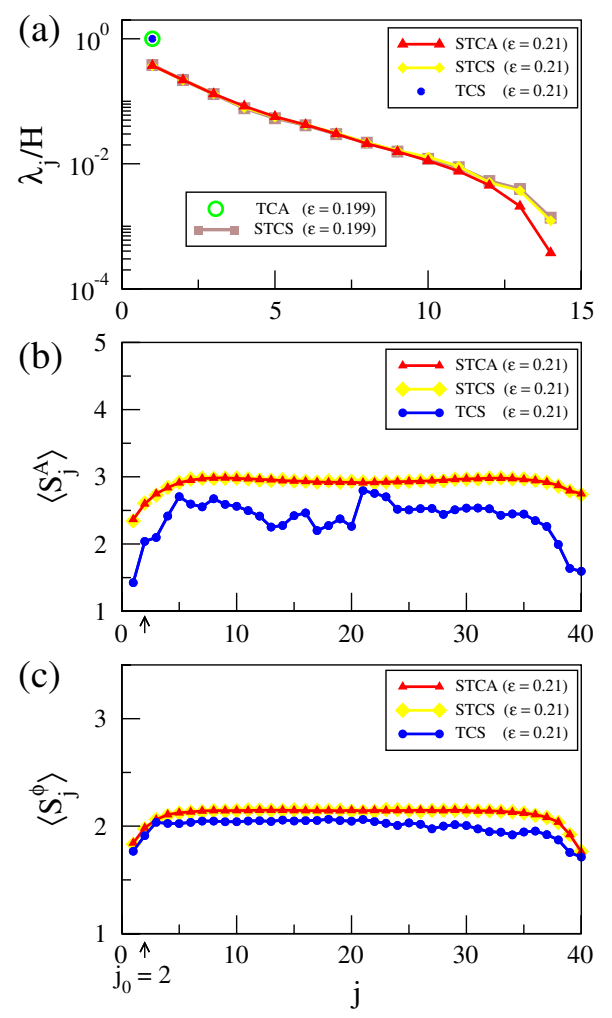

FIG. 3 (color online). Variations with the Lyapunov vector index $j$ of (a) $\lambda_{j} / H$, (b) $\left\langle S_{j}^{A}\right\rangle$, (c) $\left\langle S_{j}^{\phi}\right\rangle$. time averaging of asymptotic solutions, $\delta \hat{u}_{k}^{j}$ denotes the $k$ th Fourier component of the $j$ th Lyapunov vector, each Lyapunov vector is normalized as $\sum_{k=1}^{N}\left|\delta \hat{u}_{k}^{j}\right|^{2}=1$ for each $j$, and $\delta \phi_{k}^{j}(t)=\left(\phi_{k+1}(t)-\phi_{k}(t)\right)^{j}$ denotes the Fourier phase differences of the $j$ th Lyapunov vector. Figures 3(b) and 3(c) show the variation of $\left\langle S_{j}^{A}(t)\right\rangle$ and $\left\langle S_{j}^{\phi}(t)\right\rangle$, respectively, as a function of $j$ for STCA and the embedded STCS and TCS after the onset of permanent STC for $\varepsilon=0.21$. It is seen that for all $j$ the degree of amplitude and phase disorder of TCS is lower than both STCA and STCS. The above results of $\left\langle S_{j}^{A}(t)\right\rangle$ and $\left\langle S_{j}^{\phi}(t)\right\rangle$ are in agreement with $H$ and the Kaplan-Yorke dimension $D=p+\sum_{j=1}^{p} \lambda_{j} /\left|\lambda_{p+1}\right|, \quad p=\max \left\{m \mid \sum_{j=1}^{m} \lambda_{j} \geq 0\right\}$ computed from the Lyapunov spectrum in Fig. 3(a) [14], which yield $H=0.38(0.06)$ and $D=36.15$ (22.23) for STCS (TCS). The numerical values of $H$ and $D$ for STCA are very close to the respective values of STCS.

In the Fourier-Lyapunov representation, the timeaveraged power spectrum is defined as $\left\langle\left|\delta \hat{u}_{k}^{j}\right|^{2}\right\rangle=$ $\left\langle\left|\left(\delta u_{k}^{R}+i \delta u_{k}^{I}\right)^{j}\right|^{2}\right\rangle$ and the time-averaged phase spectrum is defined as $\left\langle\delta \phi_{k}^{j}\right\rangle=\left\langle\left(\phi_{k+1}-\phi_{k}\right)^{j}\right\rangle$. Figure 4 gives an overview of the time-averaged Fourier-Lyapunov power and phase spectra at $\varepsilon=0.21$ after the onset of permanent (a)

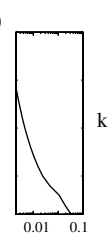

(b)

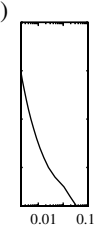

(c)

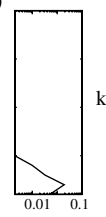

$\left\langle\left|\hat{u}_{k}\right|^{2}\right\rangle$

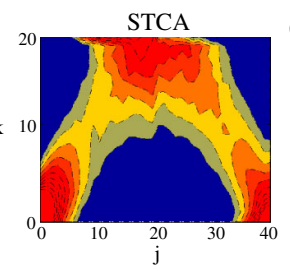

STCS

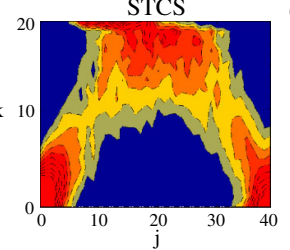

TCS

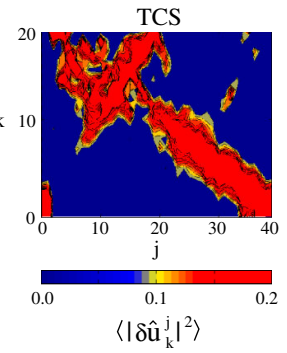

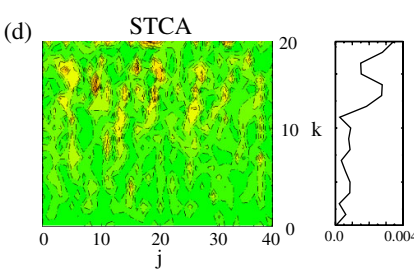
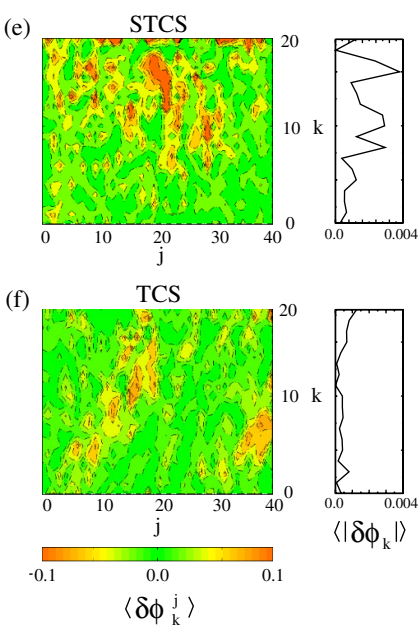

FIG. 4 (color online). On-off spatiotemporal intermittency at $\varepsilon=0.21$. Left panels: the amplitude dynamics of STCA, STCS, and TCS quantified by the time-averaged Fourier-Lyapunov power spectra $\left\langle\left|\delta \hat{u}_{k}^{j}\right|^{2}\right\rangle$. Right panels: the phase dynamics quantified by the time-averaged Fourier-Lyapunov phase spectra $\left\langle\delta \phi_{k}^{j}\right\rangle$. The side panels show the time-averaged Fourier power spectra $\left\langle\left|\hat{u}_{k}\right|^{2}\right\rangle$ and the time-averaged Fourier phase spectra $\left\langle\delta \phi_{k}\right\rangle$, respectively. 
STC, for STCA and the embedded STCS and TCS, respectively. Contour levels are indicated in the scales at the bottom.

Figure 4(c) shows that for TCS the energy is localized at small $k$ and $j$ indicated by the red patch near the origin, in agreement with Fig. 3(a) which shows that for TCS only one Lyapunov vector is growing whereas all other Lyapunov vectors are damped. In contrast, Fig. 4(b) shows that for STCS the energy that is injected into the $\kappa=k=$ 1 mode spreads to higher $k$ and $j$, and according to Fig. 3(a) 14 Lyapunov vectors are growing; similar energy spreading is seen in STCA in Fig. 4(a). The above behaviors of the time-averaged Fourier-Lyapunov power spectra of TCS, STCS, and STCA are consistent with the amplitude dynamics quantified by $\left\langle S_{j}^{A}(t)\right\rangle$ in Fig. 3(b). The left side panels of Figs. 4(a)-4(c) show the corresponding timeaveraged Fourier power spectra, which confirm that the energy bandwidth of TCS is much narrower than for STCS and STCA. Note that for both STCA and STCS the energy is confined in a narrow inverted- $V$ region in the $k-j$ space, distinct from the Gledzer-Ohkitani-Yamada shell model of fully developed turbulence where the energy is confined in a narrow $\mathrm{V}$ region [14]. This distinction arises from the difference in energy injection and dissipation of the two models.

The phase dynamics of STCA, STCS, and TCS at $\varepsilon=$ 0.21 in Figs. 4(d)-4(f) is in full accord with the amplitude dynamics in Figs. 4(a)-4(c). Figure 4(f) shows that for TCS the phase differences are small (dominance of green over yellow) across all $k$ at $j \sim 1$, associated with a higher degree of amplitude synchronization resulting from energy localization at small $k$ and $j$ seen in Fig. 4(c). In contrast, Fig. 4(e) shows that for STCS the phase differences are large (dominance of yellow over green) across all $k$ and $j$, associated with a lower degree of amplitude synchronization resulting from energy spreading to higher $k$ and $j$ seen in Fig. 4(b). The time-averaged Fourier phase spectra in the right side panels of Figs. 4(e) and 4(f) confirm that on average the phase differences across spatial scales $k$ in TCS are smaller than in STCS. A comparison of Figs. 4(d) and 4(e) shows that across all $k$ and $j$ on average the phase differences for STCS are larger than for STCA (dominance of yellow over green), implying a lower level of phase synchronization in STCS in relation to STCA; a comparison of Figs. 4(a) and 4(b) shows that across all $k$ and $j$ the pattern of Fig. 4(b) is less homogeneous than that of Fig. 4(a), displaying fewer smooth curves and more patchy patterns, implying a lower level of amplitude synchronization in STCS in relation to STCA. These features are manifestations of the combined contributions of the coexisting TCS and STCS in STCA.

In conclusion, we demonstrated the duality of amplitude and phase synchronization in an on-off spatiotemporal intermittency. The on state related to a temporally chaotic saddle is characterized by a high degree of amplitudephase synchronization, narrow bandwidth, and only one positive Lyapunov exponent, whereas the off state related to a spatiotemporally chaotic saddle is characterized by a low degree of amplitude-phase synchronization, broad bandwidth, and 14 positive Lyapunov exponents. We showed that the amplitude-phase synchronization related to multiscale interactions (Figs. 1, 3, and 4) is the origin of nonattracting coherent structures [STCS and TCS in Fig. 2(c)] embedded in the spatiotemporally chaotic attractor [STCA in Fig. 2(d)]. The methods developed in this Letter are applicable to simulated and experimental data in physical, biological, and chemical systems [5] including fully developed fluid and plasma turbulence [14,15].

This work is supported by CNPq, FAPESP, and RIMS.

*abraham.chian@gmail.com

[1] T. Tél and Y.-C. Lai, Phys. Rep. 460, 245 (2008).

[2] R. Meucci, E. Allaria, F. Salvadori, and F. T. Arecchi, Phys. Rev. Lett. 95, 184101 (2005).

[3] B. Hof, A. de Lozar, D. J. Kuik, and J. Westerweel, Phys. Rev. Lett. 101, 214501 (2008).

[4] J. D. Skufca, J. A. Yorke, and B. Eckhardt, Phys. Rev. Lett. 96, 174101 (2006).

[5] T. Klinger et al., Phys. Rev. Lett. 79, 3913 (1997); Z. L. Qu, J. N. Weiss, and A. Garfinkel, ibid. 78, 1387 (1997); Kaifen He, ibid. 94, 034101 (2005); E.L. Rempel, A. C.-L. Chian, and R. A. Miranda, Phys. Rev. E 76, 056217 (2007).

[6] E. L. Rempel and A. C.-L. Chian, Phys. Rev. Lett. 98, 014101 (2007); E. L. Rempel, R. A. Miranda, and A. C.-L. Chian, Phys. Fluids 21, 074105 (2009).

[7] T. B. Benjamin, J. L. Bona, and J. J. Mahony, Phil. Trans. R. Soc. A 272, 47 (1972).

[8] Kaifen He and A. C.-L. Chian, Phys. Rev. Lett. 91, 034102 (2003).

[9] Kaifen He and A. C.-L. Chian, Phys. Rev. E 69, 026207 (2004).

[10] G. V. Osipov et al., Phys. Rev. Lett. 91, 024101 (2003).

[11] P. Tass et al., Phys. Rev. Lett. 81, 3291 (1998); Y.-C. Lai, M. G. Frei, and I. Osorio, Phys. Rev. E 73, 026214 (2006).

[12] D. Sweet, H. E. Nusse, and J. A. Yorke, Phys. Rev. Lett. 86, 2261 (2001).

[13] H. Fujisaka and T. Yamada, Prog. Theor. Phys. 69, 32 (1983); A. S. Pikovsky and P. Grassberger, J. Phys. A 24, 4587 (1991); N. Platt, E. A. Spiegel, and C. Tresser, Phys. Rev. Lett. 70, 279 (1993).

[14] M. Yamada and K. Ohkitani, Phys. Rev. Lett. 60, 983 (1988); Phys. Rev. E 57, R6257 (1998).

[15] D. Koga, A.C.-L. Chian, R. A. Miranda, and E. L. Rempel, Phys. Rev. E 75, 046401 (2007); A. C.-L. Chian et al., Nonlinear Proc. Geophys. 15, 567 (2008); A. C.-L. Chian and R. A. Miranda, Ann. Geophys. 27, 1789 (2009). 